\title{
Isolation of a chemical inhibitor against K-Ras-induced p53 suppression through natural compound screening
}

\author{
SU-JIN LEE ${ }^{1 *}$, YOUN-SANG JUNG ${ }^{1 *}$, SUN-HYE LEE ${ }^{1}$, HYE-YOUNG CHUNG ${ }^{2}$ and BUM-JOON PARK ${ }^{1}$ \\ ${ }^{1}$ Department of Molecular Biology, College of Natural Science, ${ }^{2}$ College of Pharmacy, \\ Pusan National University, San 30, Jangjeon-dong, Geumjoung-gu, Busan, Korea
}

Received January 15, 2009; Accepted March 10, 2009

DOI: 10.3892/ijo_00000294

\begin{abstract}
The strong tumor suppressor p53 shows loss of function in large portion of human cancer. In addition to genetic mutation, biological function of p53 is suppressed by signaling distortion or elevated expression of p53 inhibitors (such as overexpression of MDM2 or deletion of p14/ARF). In this study, we demonstrate that K-Ras, a frequently altered oncogene in human cancers including pancreatic cancer (about $80 \%)$, colon cancer (45\%) and lung cancer (45\%), suppresses p53. Based on this fact, we perform Western blot analysisbased chemical screening to isolate a K-Ras-specific activator of p53. From 117 kinds of chemicals (34 kinds of natural compounds that are obtained from herbal plants, 53 kinds of flavonoid, and 31 kinds of phenolic compounds), we find that quercetin works as an activator of p53 in K-Ras mutated cells but not in wild-type cells. Treatment with quercetin can induce p53 target genes such as PUMA and p21. These results suggest that although quercetin has limitations for use as a therapeutic drug due to its broad effects, specific function of it on K-Ras-p53 may be useful for K-Ras-induced cancer prevention and therapy through further development.
\end{abstract}

\section{Introduction}

Since cancer is a genetic disease, various kinds of genetic alternations occur during tumorigenesis (activation of oncogenes and inactivation of tumor suppressors) (1). Among these cancer-related genes, p53 is an intensively studied tumor suppressor protein because of its frequent mutations in cancer $(2,3)$ and engagement in various kinds of cellular physiologies including apoptosis, cell cycle and senescence

Correspondence to: Dr Bum-Joon Park, Department of Molecular Biology, Pusan National University, San 31, Jangjeon-dong, Geumjoung-gu, Busan, Korea

E-mail: bjpark1219@pusan.ac.kr

*Contributed equally

Key words: p53, K-Ras, quercetin, chemical screening
$(4,5)$. Since cancer cells should overcome apoptosis, cell cycle inhibition, and senescence (6), p53 inactivation is prerequired event for cancer development. However, approximately $50 \%$ of cancers possess wild-type p53. How can wild-type p53 be suppressed? Expression of MDM2 has been proposed. Since MDM2 is an inhibitor of p53 (7), elevated expression of MDM2 will contribute to cancer progression (8). However, genetic amplification of MDM2 is rare event in epithelial carcinoma, despite being a strong inhibitor of p53 (9). Another plausible suggestion is deletion or silencing of $\mathrm{p} 14 / \mathrm{ARF}$, alternative splicing product of p16/INK4A (10). Since p14/ARF is an inhibitor of MDM2 (11), loss of p14/ARF suppresses p53 through MDM2. Indeed, deletion or hypermethylation of p14/ARF locus is frequently detected in human cancer (12). However, p14/ARF deficient mice show different cancer spectrum (13). These results suggest that there would be $\mathrm{p} 53$-independent tumor suppressive function in p14/ARF. In addition, wild-type p53 and p14/ARF deletion is not mutually exclusive (14). These results indicate that there would be an additional p53 suppression mechanism in human cancer development.

$\mathrm{K}-\mathrm{Ras}$ is a member of human Ras family and its active mutation is detected in about $30 \%$ of human cancer (15). In particular, pancreatic cancers as well as adenomas show extremely high mutation rate (about 80\%) (16). Considering these facts, it would be very useful for curing and preventing of human cancers such as pancreatic cancers to inhibit the oncogenic K-Ras or its function selectively.

Although cancer is a genetic disease, occurrence of cancer is obviously affected by environmental factors (17). In particular, some kinds of foods and nutrients show tumorprevention effect. A well known example is the Frenchparadox (18). Despite a high fat diet, a low incidence of colon cancer in the French is due to continuous uptake of resveratrol, component of red wine (19). Resveratrol exerts an anti-inflammatory and anti-oxidant factor and reduces the chronic inflammation that is one of well known causes of colon cancer (20). This fact implies that through dairy diet, we can prevent cancer partially and also indicates that there would be other kinds of anti-cancer or cancer preventing factors in natural compounds.

In this study, we revealed that oncogenic K-Ras could suppress p53 function. Based on this knowledge, we isolated a specific inhibitor of oncogenic K-Ras-mediated p53 suppression from natural compounds. 

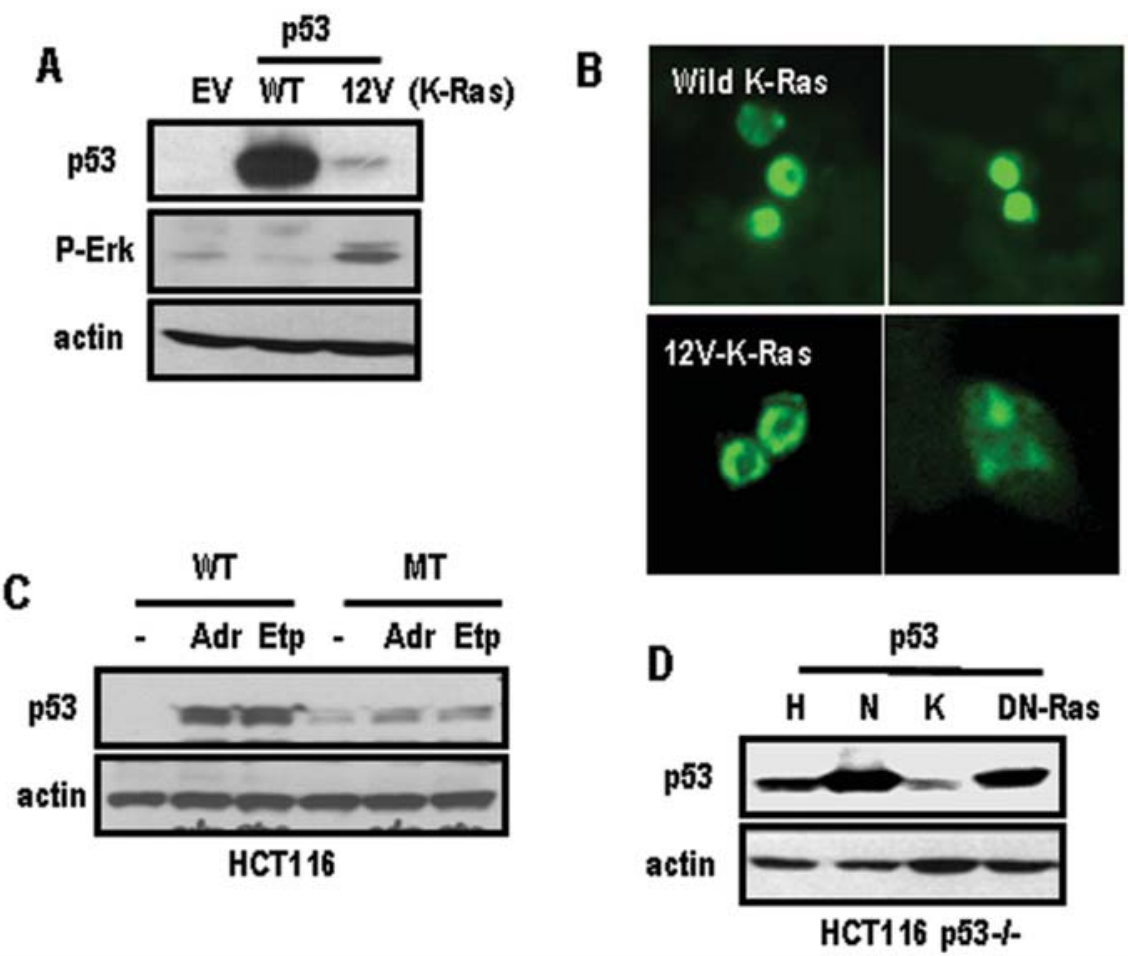

Figure 1. Oncogenic K-Ras suppresses p53. A, Transfected p53 into HCT116 p53 ${ }^{--}$cells was obviously suppressed by oncogenic K-Ras but not wild-type K-Ras. B, Immunostaining analysis also showed the same result. C, Oncogenic K-Ras also suppresses DNA-Damage-induced p53 activation. HCT116 p53 ${ }^{+}$ cells were incubated with (adriamycin) Adr $(0.2 \mu \mathrm{g} / \mathrm{ml})$ and (etoposide) Etop $(10 \mu \mathrm{M})$ for $2 \mathrm{~h}$. D, Comparing to other kinds of Ras, p53 suppression ability is a unique function of K-Ras. HCT116 p53\%- cells were co-transfected with p53 and Ras or DN-Ras.

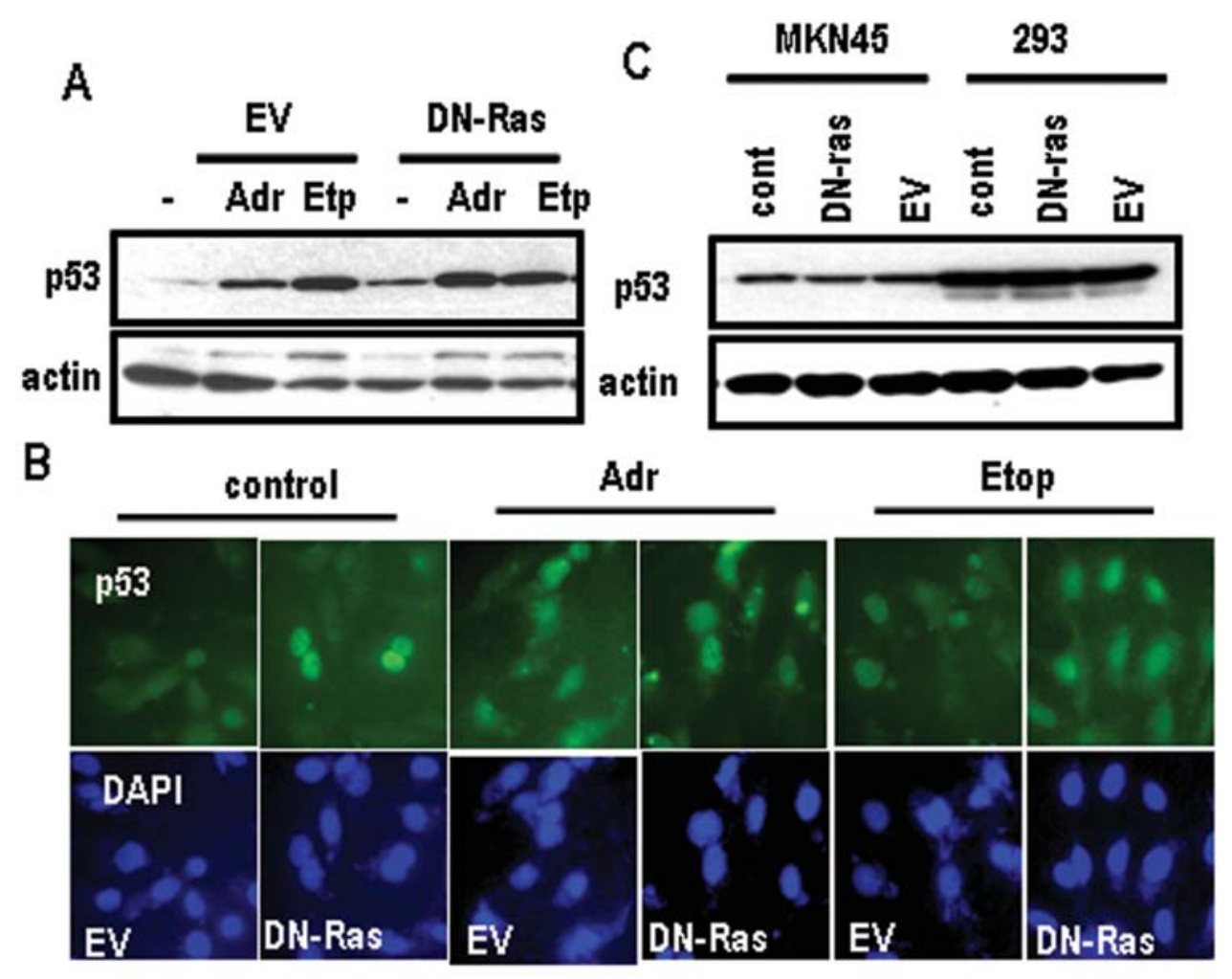

Figure 2. Suppression of endogenous K-Ras can induce p53. A, Blocking of Ras activity through DN-Ras can increase p53 expression in A549 and also improves sensitivity of p53 in response to DNA damage. Since Etop show saturated effect, we did not observe additional induction of p53 in DN-Ras+Etop (lane 6). B, Immunostaining also shows the increase of p53 expression by DN-Ras. Green (FITC) indicates p53. Staining of DAPI (blue) was performed to visualize the nucleus. C, However, K-Ras wild-type cells did not show induction of p53 by DN-Ras. MKN-45 and 293 cells were transfected with vehicle (control), DNRas or empty vectors. 


\section{Materials and methods}

Cell culture and reagents. Cell lines (HCT116, A549, MKN-45 and MCF-7) were obtained from ATCC and maintained in culture media (RPMI-1640 or DMEM) supplemented with $10 \%$ FBS and $1 \%$ antibiotics. p53 deficient HCT116 was provided by B. Vogelstein (Johns-Hopkins). Antibodies against p53 (DO-1) and actin were purchased from Santa Cruz Biotech, and p-Erk, PUMA, and PARP were obtained from Calbiochem. Chemicals (natural compounds, flavonoids, and phenolic compounds) were kindly provided by Korean Aging Tissue Bank in Pusan National University (full chemical list is available upon request). General chemicals were obtained from Sigma.

Transfection and vectors. Three kinds of oncogenic Ras vectors (all of them possess mutation at codon 12V) and DN-Ras (21) were kindly provided by S.G. Chi (Korea University). p53 expression vector has been described previously (22). For transfection, cells were incubated with vector and Jetpei mixture for $3 \mathrm{~h}$ in $1 \mathrm{ml}$ in serum-free conditions. Equal volume of $20 \%$ FBS containing medium was added to cells. After $24 \mathrm{~h}$, cells were harvested and subjected to Western blot analysis or immunostaining analysis.

Western blot analysis. Cells transfected with indicated vectors or treated with chemicals, were lyzed with RIPA and denatured by boiling with sample buffer. For separation of proteins, $20 \mu \mathrm{g}$ sample was loaded in each lane. After electrophoresis, proteins were transferred to PVDF membrane using semi-dry transfer kit (Bio-Rad). After blocking with 3\% non-fat dry milk in TBST, membranes were incubated with primary $\mathrm{Ab}$ in blocking buffer (TBST $+1 \%$ milk) and HRT-conjugated secondary $\mathrm{Ab}$. Reacted Ab was detected by ECL solution (Intron) and exposed to X-ray film.

Immunostaining. To examine the expression and localization of p53, cells, transfected with p53 or DN-Ras, were fixed with $100 \% \mathrm{Me}-\mathrm{OH}$ for $10 \mathrm{~min}$ at $-20^{\circ} \mathrm{C}$ and incubated with blocking buffer (PBS $+1 \%$ BSA + normal goat IgG). After washing with ice-cold PBS twice, cells were incubated with primary (DO-1, 1:500) and FITC-conjugated secondary Ab (1:1000) for $2 \mathrm{~h}$. After washing, reacted $\mathrm{Ab}$ was visualized by fluorescence microscopy.

\section{Results}

K-Ras suppresses p53. To examine the effect of K-Ras on p53, we transfected oncogenic K-Ras and wild-type K-Ras into

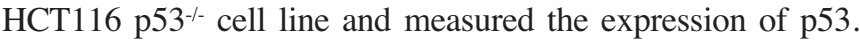
Forced expression of oncogenic K-Ras obviously suppressed the p53 expression (Fig. 1A). We also observed the reduction of p53 through immunostaining (Fig. 1B). K-Ras also blocked the activation of p53 in response to DNA damage signaling (Fig. 1C). To determine whether p53 suppression was specific to K-Ras or a general effect of oncogenic Ras, we co-transfected p53 with $\mathrm{H}-, \mathrm{K}-$, and N-Ras and measured the expression of p53. Interestingly, only K-Ras showed the p53 suppression effect (Fig. 1D).
A

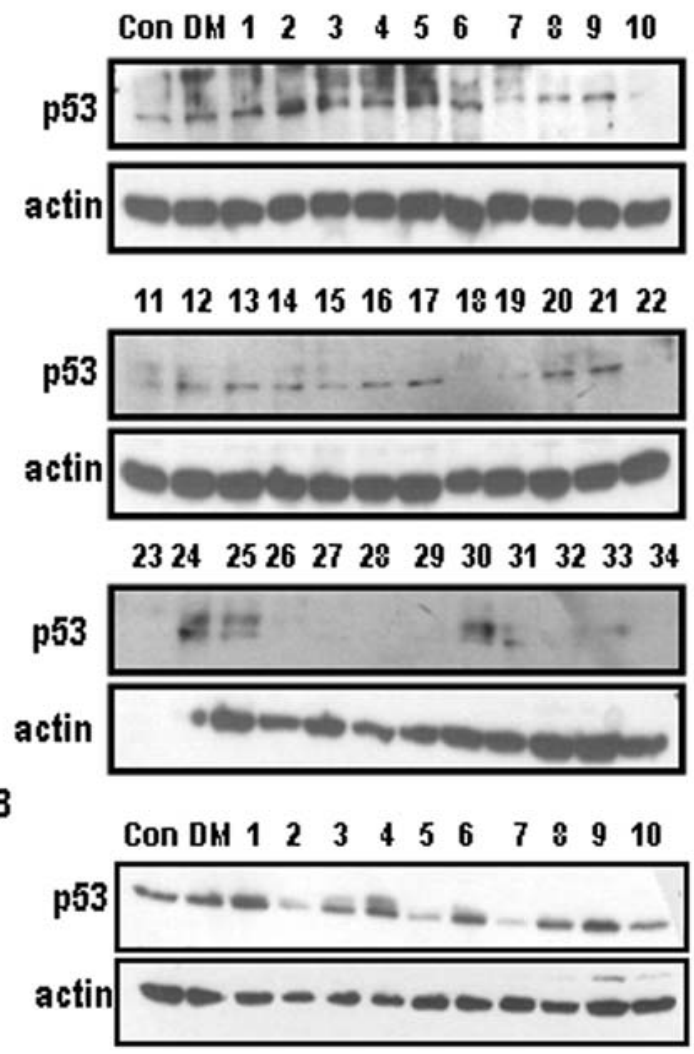

111213141516171819202122

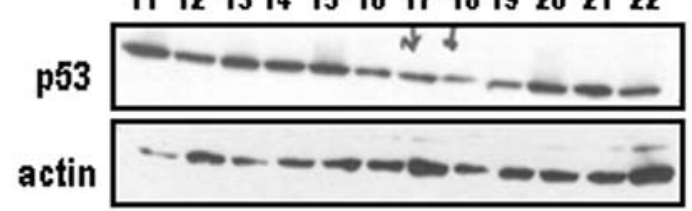

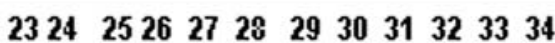

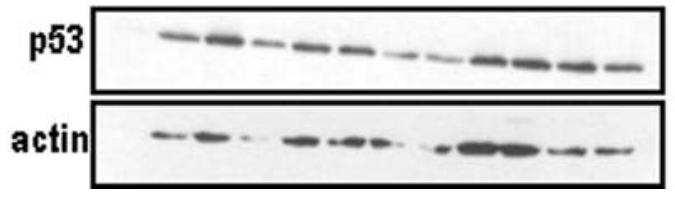

Figure 3. Screening of natural compounds by Western blot analysis. A, To isolate the p53 activator, A549 cells were incubated with 34 kinds of chemicals $(10 \mu \mathrm{M})$ for $2 \mathrm{~h}$. Cells were lyzed with RIPA and subjected to SDSPAGE and WB analysis. Actin was used for loading control. Some chemicals showed cytotoxicity so that expression of all kinds of proteins disappeared (nos. 23 and 24). B, Using the same chemicals, MKN-45 was also treated under the same condition. Nos. 23 and 24 also showed toxicity. Comparing two blots, nos. 2, 5, 12, 24, SAL-2 showed p53 induction activity only in A549.

Suppression of K-Ras activity induces p53 expression. To investigate the effect of endogenous mutant K-Ras, we blocked the Ras activity through DN (dominant negative)Ras and measured the expression of p53 in A549 cells (KRas mutated cell line). Transfection of DN-Ras induced p53 expression and sensitivity to adriamycin (Adr) (Fig. 2A). We confirmed it through immunostaining analysis (Fig. 2B). However, MKN45 and 293 cell lines (wild-type K-Ras) did not responded to DN-Ras (Fig. 2C). These results indicated that endogenous mutant K-Ras also suppressed p53 expression. In addition, these results imply that specific 

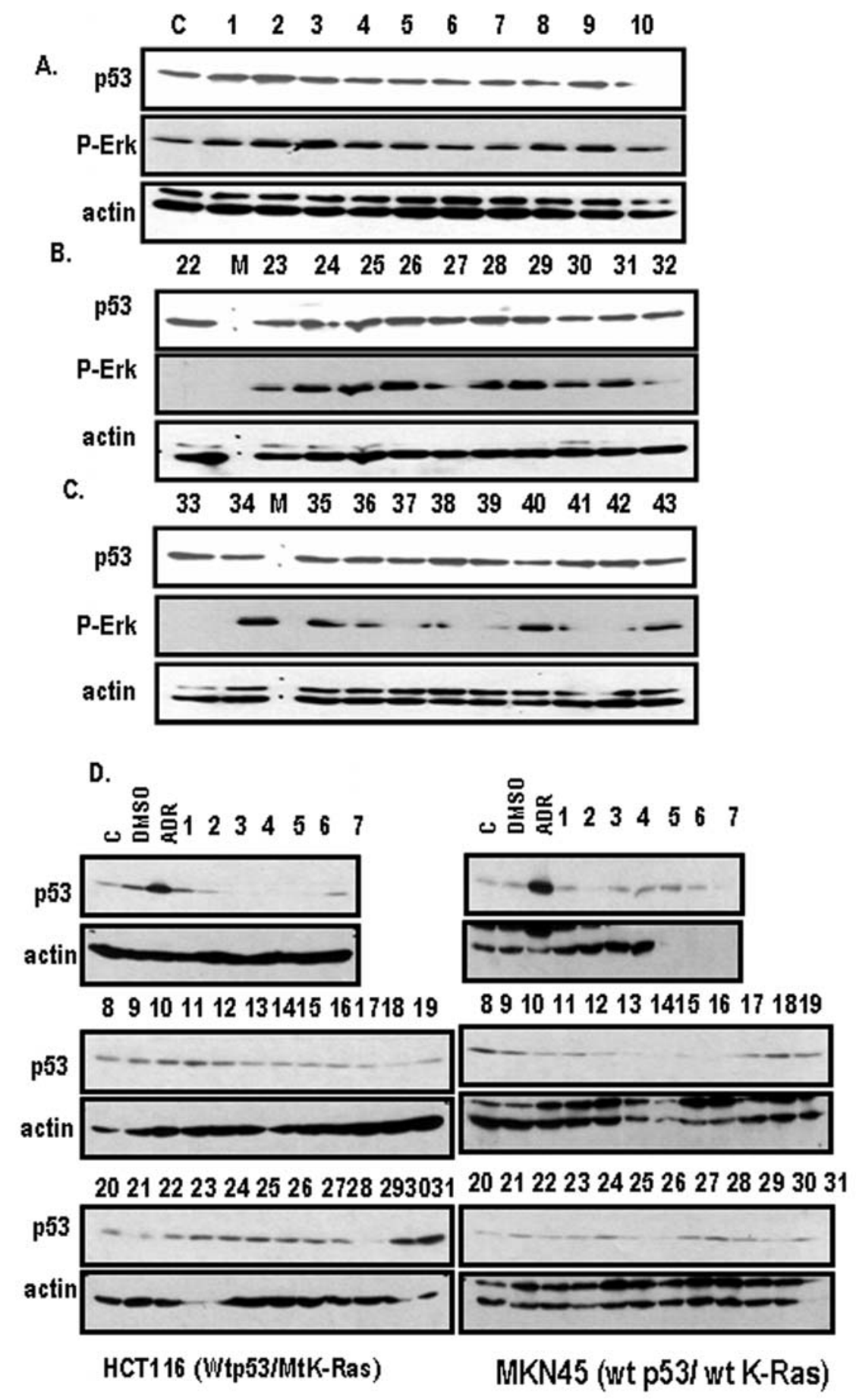

Figure 4. Screening of flavonoids and phenolic compounds. Using the same conditions, we performed screening of flavonoids (A-C) and phenolic compounds (D). However, the chemicals did not show p53 induction ability. Although nos. 30 and 31 of phenolic compounds showed the effect on p53, from our careful re-analysis, their effect was not reproducible.

inhibition of oncogenic K-Ras may be a useful strategy for KRas-induced human cancers such as pancreatic cancer, lung cancer and colon cancer (23). Our result is also consistent with other reports that elimination of oncogenic K-Ras can induce p53 expression (24).

Screening of natural compounds. To isolate the specific inhibitor on K-Ras-mediated p53 suppression, we first screened the natural compounds, which are extracted from herbal plants or vegetables. Rational basis of our strategy (why we chose the extract of herbal plants or vegetables as initial candidates) are: i) occurrence of pancreatic cancer and colon cancer is quite different between Western and Asia, ii) Asian main dairy diet is vegetables, and iii) in fact, pancreatic and colon cancer incidence is dramatically increased in Korea, consistently with change of diet pattern from vegetables to high fat and high calorie diets (25). Among 34 kinds of natural compounds, some of them induced p53 expression in A549 cells (Fig. 3A). In contrast, others showed cytotoxicity. Majority of them did not show obvious effect in our conditions. Since our goal was the isolation of K-Rasdependent p53 inducer, we measured the p53 again in 

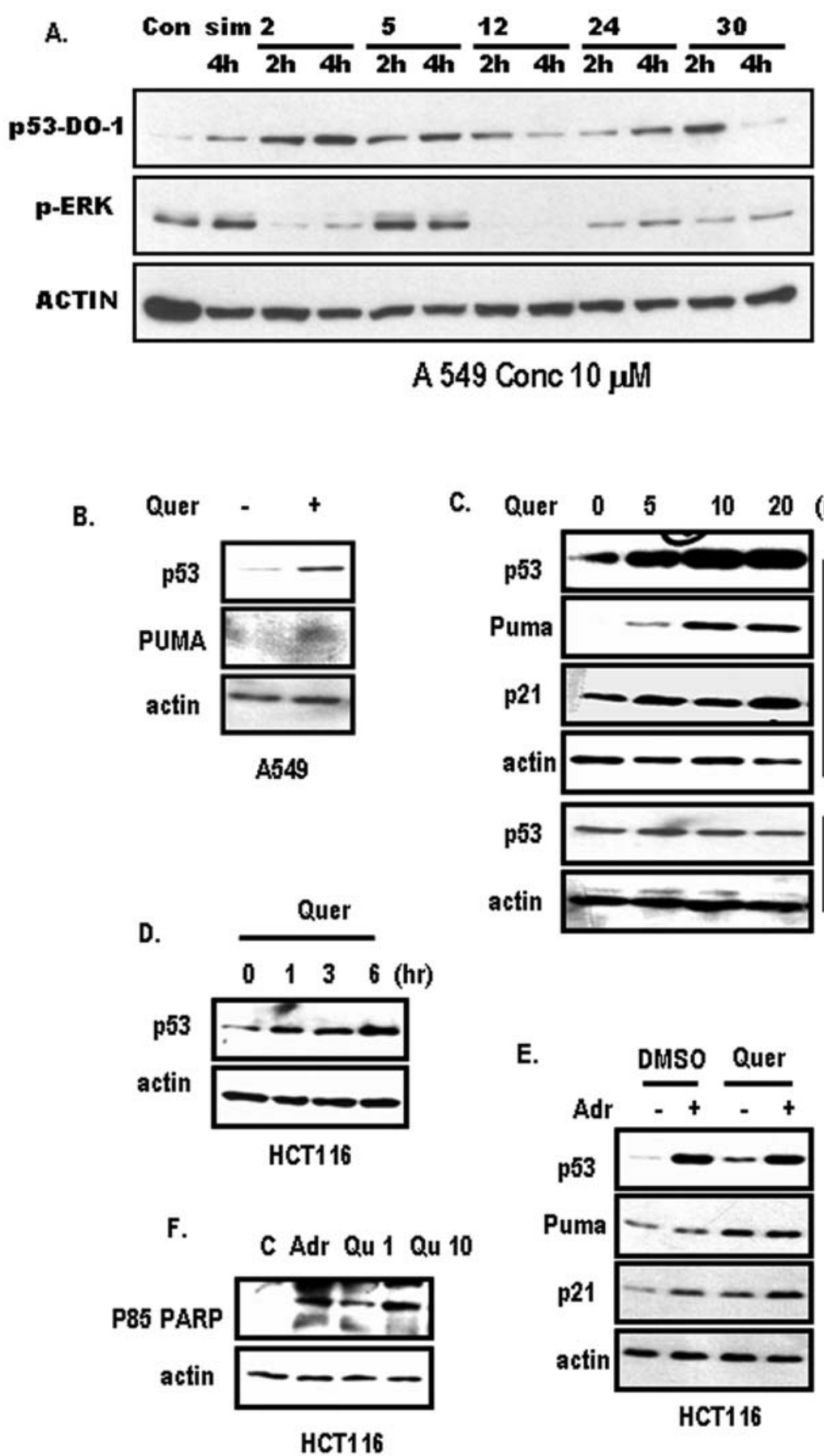

C. Quer $\begin{array}{llllll} & 5 & 5 & 10 & 20 & (\mathrm{mM})\end{array}$

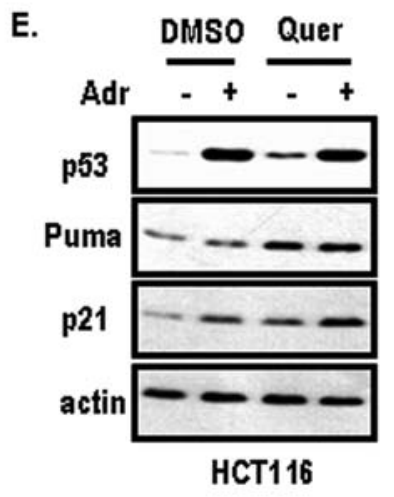

Figure 5. Effect of quercetin on the p53 pathway. A, To confirm the effect of chemicals, obtained from natural compound screening, we examined the expression of p53 and p-Erk again in A549. Although 5 chemicals could induce p53, no. 2 (quercetin) shows obvious effect on induction of p53. Concentration of each chemical was $10 \mu \mathrm{M}$. B, Treatment of quercetin can induce p53 as well as its target gene, PUMA. C, Quercetin-induced p53 is also detected in other kind of K-Ras mutated cell line, HCT116 dose-dependently. However, this chemical can not induce p53 in MCF-7 (wild-type K-Ras cell line). D, Time-dependent induction of p53 is also observed in HCT116. E, Production of cleaved PARP (p85) can be detected by quercetin treatment, suggesting that quercetin can induce apoptosis in A549. $\mathrm{F}$, Quercetin shows a synergic effect with adriamycin on expression of p21. Although quercetin alone did not induce p53 as strong as Adr, induction of target genes (p21 and PUMA) was obviously detected. Expression of p21 was induced when Adr and quercetin were combined.

MKN45 (wild-type K-Ras cell line). In this assay, we also observed the changes of $\mathrm{p} 53$ expression in response to certain chemicals (Fig. 3B). Comparing both results, we identified 5 chemicals $(2,5,12,24$, and SAL-2) as K-Ras-dependent p53 activators. In contrast, some chemicals induced p53 regardless of K-Ras status $(11,20,21,25)$ and suppressed p53 (7, 10, and GGTI). These results strongly suggested that some kinds of natural compounds can exert a protective effect against cancer and others can induce toxicity.
Screening of flavonoids and phenolic compound. To extend our screening, we obtained additional flavonoid chemicals and phenolic compounds from Korean Aging Tissue Banks and screened through the same method. We examined the 53 kinds of flavonoids. However, we could not find a p53 inducer in A549 cells (data not shown). Next, we screened 31 kinds of phenolic compounds. But in this case, we did not find useful candidates (Fig. 4). These results suggested that our findings were not artifacts. 
Quercetin activates p53 and induces its target genes. Since we did not obtain other candidates from screening of flavonoids and phenolic compounds, we confirmed the effect of our previous chemicals, selected from natural compounds screening. Since our final goal was to obtain a K-Rasspecific p53 activator, we compared the effect of these chemicals on p53 induction in A549 and MKN45. Although all of them could induce p53 in a K-Ras-dependent manner, the effect of quercetin (no. 2) was better than that of others. Quercetin showed time-dependent induction of p53 and also suppression of p-Erk (Fig. 5A). Since p53 is a transcriptional factor and its tumor suppressive role is achieved through induction of target genes, we measured the effect of quercetin on expression of PUMA, immediate target of p53 and strong inducer of apoptosis $(26,27)$. Treatment with quercetin induced PUMA in A549 cells (Fig. 5B). To confirm the dependence of oncogenic K-Ras, we investigated this chemical in HCT116 (K-Ras mutated colon cancer cell) and MCF-7 cells (wild-type Ras-breast cancer cell line), and found that quercetin could induce p53 and its target genes (p21 and PUMA) only in HCT116, but not in MCF-7 (Fig. $5 \mathrm{C})$. Next we measured the time-dependent induction of $\mathrm{p} 53$. Induction of p53 in response to quercetin was detected within $1 \mathrm{~h}$ and maintained for $6 \mathrm{~h}$ (Fig. 5D). As expected, this chemical evoked apoptosis in A549, which was monitored by production of cleaved PARP (p85; Fig. 5E). Finally, we checked the synergic effect of quercetin with the well known anti-cancer drug adriamycin. Although adriamycin alone could not induce PUMA, quercetin could induce PUMA in A549 (Fig. 5F). Moreover, co-treatment of adriamycin and quercetin could obviously induce p21 expression (Fig. 5F). This result was consistent with our previous results that DN-Ras enhanced the sensitivity to adriamycin-induced p53 (Fig. 2A).

\section{Discussion}

We show that quercetin can induce p53 in a K-Rasdependent manner. Quercetin is a natural compound found in grapes and onions. It has been reported that long-term uptake of quercetin can reduce cancer incidence (28). However, its clear molecular working mechanism has not been proposed. In this study, we reveal that quercetin possesses p53 activating potential that is fully dependent on K-Ras mutation. Although we do not know the molecular target of quercetin, because of the ambiguous molecular mechanism on K-Ras-mediated p53 suppression, the quercetin effect on p53 seems to be reliable.

This fact is also consistent with previous results that cancer prevention effect of quercetin is clearly detected in pancreatic and colon cancer, because both kinds of cancer are initiated by a K-Ras mutation (29). However, a natural compound is not a drug so that an over-dose would evoke unexpected effects. We did observe an unexpected GSK-3B inhibition by higher dose-quercetin (data not shown). Since GSK-3ß inhibition can induce activation of ß-catenin (30) and promote colon cancer (31), proper range of quercetin may show cancer prevention effects. When the molecular mechanism of K-Ras-mediated p53 suppression and molecular target of quercetin become clear, quercetin may be a useful compound for development as a anti-cancer drug for pancreatic and colon cancer.

In conclusion, we revealed that oncogenic K-Ras suppresses p53 and suggest that it may be one of p53 inactivated mecha-nism in human cancer. Moreover, inhibitor of K-Ras-mediated p53 suppression may be a useful target for anti-cancer drugs. Our chemical screening analysis shows that quercetin can induce p53 in a K-Ras-dependent manner. Thus, quercetin may be a compound suitable for further development as an anti-cancer drug.

\section{Acknowledgements}

We specially thank Dr S.G. Chi for Ras vectors and DN-Ras vector, members of Korea Aging Tissue Bank for chemicals, and other members of our laboratory. This work was supported by a 2-year research fund of Pusan National University (2006-2008).

\section{References}

1. Hanahan D and Weinberg RA: The hallmarks of cancer. Cell 100: 57-70, 2000

2. Vogelstein B, Lane B and Levine AJ: Surfing the p53 network. Nature 408: 307-310, 2000.

3. Sherr CJ: Principle of tumor suppression. Cell 116: 235-246, 2004.

4. Dimri GP: What has senescence got to do with cancer? Cancer Cell 7: 505-512, 2005.

5. Levine AJ: p53, the cellular gatekeeper for growth and division. Cell 88: 323-331, 1997.

6. Lowe SW, Cepero E and Evan G: Intrinsic tumor suppression. Nature 342: 307-315, 2004.

7. Brooks CL and Gu W: p53 ubiquitination: $\mathrm{Mdm} 2$ and beyond. Mol Cell 21: 307-315, 2006

8. Michael D and Oren M: The p53-Mdm2 module and the ubiquitin system. Semin Cancer Biol 13: 49-59, 2003.

9. Bond GL, Hu W, Bond EE, Robins H, Lutzker SG, Arva NC, Bargonetti J, Bartel F, Taubert H, Wuerl P, Onel K, Yip L, Hwang SJ, Strong LC, Lozano G and Levine AJ: A single nucleotide polymorphism in the MDM2 promoter attenuates the p53 tumor suppressor pathway and accelerates tumor formation in humans. Cell 119: 591-602, 2004.

10. Weber JD, Kuo ML, Bothner B, DiGiammarino EL, Kriwacki RW, Roussel MF and Sherr CJ: Cooperative signals governing ARFmdm2 interaction and nucleolar localization of the complex. Mol Cell Biol 20: 2517-2528, 2000.

11. Stott FJ, Bates S, James MC, McConnell BB, Starborg M, Brookes S, Palmero I, Ryan K, Hara E, Vousden KH and Peters G: The alternative product from the human CDKN2A locus, p14(ARF), participates in a regulatory feedback loop with p53 and MDM2. EMBO J 17: 5001-5014, 1998

12. Sherr CJ and Weber JD: The ARF/p53 pathway. Curr Opin Genet Dev 10: 94-99, 2000.

13. Sherr CJ: Divorcing ARF and p53: an unsettled case. Nat Rev Cancer 6: 663-673, 2006.

14. Lowe SW and Sherr CJ: Tumor suppression by Ink4a-Arf: progress and puzzles. Curr Opin Genet Dev 13: 77-83, 2003.

15. Downward J: Targeting RAS signalling pathways in cancer therapy. Nat Rev Cancer 3: 11-22, 2003.

16. Vogelstein B, Fearon ER, Kern SE, Hamilton SR, Preisinger AC, Nakamura Y and White R: Allelotype of colorectal carcinomas. Science 214: 207-211, 1989.

17. Peto J: Cancer epidemiology in the last century and the next decade. Nature 411: 390-395, 2001.

18. Sun AY, Simonyi A and Sun GY: The 'French Paradox' and beyond: neuroprotective effects of polyphenols. Free Radic Biol Med 32: 314-318, 2002.

19. Hsieh TC, Juan G, Darzynkiewicz Z and Wu JM: Resveratrol increases nitric oxide synthase, induces accumulation of p53 and p21(WAF1/CIP1), and suppresses cultured bovine pulmonary artery endothelial cell proliferation by perturbing progression through S and G2. Cancer Res 59: 2596-2601, 1999. 
20. Chapkin RS, McMurray DN and Lupton JR: Colon cancer, fatty acids and anti-inflammatory compounds. Curr Opin Gastroenterol 23: 48-54, 2007

21. Park JI, Lee MG, Cho K and Chi SG: Transforming growth factor-betal activates interleukin-6 expression in prostate cancer cells through the synergistic collaboration of the Smad2, p38-NF-kappaB, JNK, and Ras signaling pathways. Oncogene 22: 4314-4332, 2003.

22. Park BJ, Kang JW, Lee SW and Kim S: The haploinsufficient tumor suppressor p18 upregulates p53 via interactions with ATM/ATR. Cell 120: 209-221, 2005.

23. Rowinsky EK, Windle JJ and von Hoff DD: Ras protein farnesyltransferase: A strategic target for anticancer therapeutic development. J Clin Oncol 17: 3631-3652, 1999.

24. Halaschek-Weiner J, Wacheck V, Kloog Y and Jansen B: Ras inhibition leads to transcriptional activation of p53 and downregulation of $\mathrm{Mdm} 2$; two mechanisms that cooperatively increase p53 function in colon cancer cells. Cell Signal 16: 1319-1327, 2004.
25. McCracken M, Olsen M, Chen MS Jr, Jemal A, Thun M, Cokkinides V, Deapen D and Ward E: Cancer incidence, mortality, and associated risk factors among Asian Americans of Chinese, Filipino, Vietnamese, Korean, and Japanese ethnicities. CA Cancer J Clin 57: 190-205, 2007.

26. Nakano K and Vousden KH: PUMA, a novel proapoptotic gene, is induced by p53. Mol Cell 7: 683-694, 20001.

27. Yu J, Zhang L, Hwang PH, Kinzler KW and Vogelstein B: PUMA induces the rapid apoptosis of colorectal cancer cells. Mol Cell 7: 673-682, 2001.

28. Murakami A, Ashida $\mathrm{H}$ and Terao J: Multitargeted cancer prevention by quercetin. Cancer Lett 269: 315-325, 2008.

29. Nöthlings U, Murphy SP, Wilkens LH, Henderson BE and Kolonel LN: Flavonols and pancreatic cancer risk: the multiethnic cohort study. Am J Epidemiol 166: 924-931, 2007.

30. Polakis P: Wnt signaling and cancer. Genes Dev 14: 1837-1851, 2000.

31. Clever H: Wnt breakers in colon cancer. Cancer Cell 5: 5-6, 2004. 